\title{
A Lipschitz Stability Estimate for the Inverse Source Problem and the Numerical Scheme
}

\author{
Xianzheng Jia \\ Shandong University of Technology, Shandong 255000, China \\ Correspondence should be addressed to Xianzheng Jia; jxz_1@hotmail.com
}

Received 4 May 2016; Accepted 27 June 2016

Academic Editor: Soheil Salahshour

Copyright (C) 2016 Xianzheng Jia. This is an open access article distributed under the Creative Commons Attribution License, which permits unrestricted use, distribution, and reproduction in any medium, provided the original work is properly cited.

We consider the inverse source problem for heat equation, where the source term has the form $f(t) \phi(x)$. We give a numerical algorithm to compute unknown source term $f(t)$. Also, we give a stability estimate in the case that $f(t)$ is a piecewise constant function.

\section{Introduction to the Problem}

The inverse source problems in heat equation are solving the heat equation with unknown heat sources. They are well known to be ill-posed. There were many studies to identify different types of heat sources. For example, Cannon and DuChateau [1] estimated the nonlinear temperature dependent heat source. In $[2,3]$, the method of fundamental solutions has been presented for an inverse time-dependent heat source problem. In [4-10], several numerical schemes have been proposed to determine a space-dependent heat source. In [11-13], these numerical methods were considered to solve some two- and three-dimensional inverse heat source problems. In Savateev [14], Trong et al. [15], and Shidfar et al. [16], the authors considered the source as a function of both space and time, but it was additive or separable.

Throughout this paper, we will assume that $\Omega$ is an open set in $R^{n}$. We consider the following initial-boundary value problem:

$$
\begin{aligned}
u_{t} & =\Delta u+f(t) \phi(x) \quad(x, t) \in \Omega \times(0, T), \\
u(x, 0) & =0 \quad x \in \Omega, \\
u(x, t) & =0 \quad(x, t) \in \partial \Omega \times(0, T) .
\end{aligned}
$$

Problems of the above equations include inverse problems, heat conduction processes, hydrology, material sciences, and heat transfer problems. In the context of heat conduction and diffusion, $u$ represents either temperature or concentration. In this system, $\phi(x)$ is spatial density and unknown $f(t)$ is interpreted as either a heat or a material source, respectively, in a chemical or a biochemical application.

When $f(t)>0$, it means that term $f(t) \phi(x)$ is a source. We assume that $\phi$ is a given function and satisfies the following condition:

$$
\phi \geq 0, \quad \not \equiv 0 \text { in } \Omega
$$

$\phi$ has compact support in $\Omega$.

Our problem is to derive $f(t)$ and a conditional stability in the determination of $f(t), 0 \leq t \leq T$, from the observation

$$
u\left(x_{0}, t\right), \quad 0<t<T
$$

where $x_{0} \in \Omega / \overline{\operatorname{supp} \phi}$.

\section{Some Existing Results}

To derive the stability estimate, we need the following inequalities, which can be found in [17]. Here, we just list the result.

Theorem 1. Let $p \geq 1, \delta>0,0 \leq \alpha<T$, and $f, g \in L^{\infty}(0, T)$ satisfy

$$
0 \leq f, g \leq M<\infty, \quad 0<t<T .
$$


Then,

$\|f\|_{L^{p}(\alpha, T)}\|g\|_{L^{p}(0, \delta)}$

$$
\leq M^{(2 p-2) / p}\left(\int_{\alpha}^{T+\delta}\left(\int_{\alpha}^{t} f(s) g(t-s) d s\right) d t\right)^{1 / p} .
$$

In particular, for

$$
(f * g)(t)=\int_{0}^{t} f(t-s) g(s) d s, \quad 0<t<T
$$

and for $\alpha=0$, one has

$$
\|f\|_{L^{p}(\alpha, T)}\|g\|_{L^{p}(0, \delta)} \leq M^{(2 p-2) / p}\|f * g\|_{L^{1}(0, T+\delta)}^{1 / p} .
$$

In [17], by using the above theorem, a Hölder estimate was obtained for the case that $\Omega=R^{n}$ and $f \in U$, where

$$
\begin{aligned}
\mathcal{U} & =\left\{f \in C[0, T] ;\|f\|_{C[0, T]}\right. \\
& \leq M, f \text { changes the signs at most } N \text {-times }\} .
\end{aligned}
$$

Theorem 2. Let $\phi$ satisfy (2), and set $\mathcal{U}$ to be defined as above definition.

Then, for arbitrarily given $\delta>0$, there exists a constant $C=C\left(x_{0}, \phi, T, p, \delta, \mathcal{U}\right)>0$ such that

$$
\|f\|_{L^{p}(0, T)} \leq C\left\|u\left(x_{0}, \cdot\right)\right\|_{L^{1}(0, T+\delta)}^{1 / p^{N}},
$$

for any $f \in \mathcal{U}$.

Corollary 3. In Theorem 2, if one replaces $\mathcal{U}$ by

$$
\mathscr{P}_{N}=\{f ;
$$

$f$ is a polynomial whose order is at most $N$,

$$
\left.\|f\|_{C[0, T]} \leq M\right\}
$$

also the same result can be obtained.

But we are more interested in the case that $f(t)$ is a piecewise constant function. In that case, $f(t)$ can have infinity number of zeros. For that case, we obtained the similar result, and we will claim the result and the proof in the next section.

\section{The Estimate}

For problem (1), we can see that solution $u(x, t)$ can be expressed in the following form:

$$
\begin{array}{r}
u(x, t)=\int_{0}^{t} \int_{\Omega} K(x, y, t-s) f(s) \phi(y) d y d s, \\
x \in \Omega, t>0,
\end{array}
$$

where $K(x, y, t)$ is the fundamental function for problem (1). See [18].
Also from [18], we can see that fundamental solution $K(x, y, t)$ satisfies the following conditions:

$$
K(x, y, t)>0, \quad t>0 .
$$

Therefore, setting

$$
\mu_{x_{0}}(t)=\int_{\Omega} K\left(x_{0}, y, t\right) \phi(y) d y, \quad t>0
$$

we have

$$
u\left(x_{0}, t\right)=\int_{0}^{t} \mu_{x_{0}}(t-s) f(s) d s, \quad 0<t<T
$$

which is a Volterra integral equation of the first kind with respect to $f$.

Here, we consider the case that $f \in F=$ $\{f$ is a piecewise constant function in $[0, T]\}$.

Theorem 4 (uniqueness). For problem (1), given $u\left(x_{0}, t\right)$, then $f \in F$ is uniquely determined except for finite point in $(0, T)$.

Proof. Suppose that there are two functions $f_{1}(t), f_{2}(t) \in F$ satisfying (1). We assume that the discontinuous points of $f_{1}$ and $f_{2}$ are $0=t_{0}^{1}<t_{1}^{1}<\cdots<t_{I_{1}}^{1}=T$ and $0=t_{0}^{2}<t_{1}^{2}<$ $\cdots<t_{I_{2}}^{2}=T$, respectively, and $f_{1}(t)=f_{j}^{1}, t \in\left(t_{j-1}^{1}, t_{j}^{1}\right]$ and $f_{2}(t)=f_{j}^{2}, t \in\left(t_{j-1}^{2}, t_{j}^{2}\right]$.

After some resorting procedure, we get points $0=\tau_{0}<$ $\tau_{1}<\cdots<\tau_{I_{3}}=T$; then, for $0<t<\tau_{1}$,

$$
\begin{aligned}
& \int_{0}^{t} \mu_{x_{0}}(t-s)\left(f_{1}(s)-f_{2}(s)\right) d s=u\left(x_{0}, t\right)-u\left(x_{0}, t\right) \\
& \quad=0
\end{aligned}
$$

$$
\left(f_{1}^{1}-f_{1}^{2}\right) \int_{0}^{t} \mu_{x_{0}}(t-s) d s=0
$$

and $\int_{0}^{t} \mu_{x_{0}}(t-s) d s>0,0<t<\tau_{1}$, so we get $f_{1}(t)=f_{2}(t)$, $0<t<\tau_{1}$.

For $\tau_{1}<t<\tau_{2}$

$$
\begin{aligned}
\int_{0}^{t} \mu_{x_{0}}(t-s)\left(f_{1}(s)-f_{2}(s)\right) d s \\
\quad=\int_{\tau_{1}}^{t} \mu_{x_{0}}(t-s)\left(f_{1}(s)-f_{2}(s)\right) d s=0,
\end{aligned}
$$

therefore, $f_{1}(t)=f_{2}(t), t \in\left(\tau_{1}, \tau_{2}\right)$.

Repeating the above procedure, we can get $f_{1}(t)=$ $f_{2}(t), t \in\left(\tau_{j-1}, \tau_{j}\right), j=1,2, \ldots, I_{3}$.

Thus, end the proof.

Suppose that $f$ changes its values at $0=t_{0}<t_{1}<t_{2}<$ $\cdots<t_{I}=T, I \leq N$, and $f(t)=f_{j}, t \in\left[t_{j-1}, t_{j}\right), j=$ $1,2, \ldots, I$. Here, we assume that there exists a constant $c>0$, such that $t(i)-t(i-1) \geq c, i=1,2, \ldots, I$.

Theorem 5. If $\phi$ satisfies (2), $x_{0} \in \Omega / \overline{\operatorname{supp} \phi}$. 
Then, for $p>1$, there exists a constant $C=C\left(x_{0}, \phi, T\right.$, $p, F, N)>0$ such that

$$
\|f\|_{L^{p}(0, T)} \leq C\left\|u\left(x_{0}, \cdot\right)\right\|_{L^{p}(0, T)},
$$

for any $f \in F$. Here, $N$ is the number of intervals in $[0, T]$ that $f \neq 0$.

Proof. Since $\mu_{x_{0}}(t)$ is positive and bounded and (13), we can see that

$$
u\left(x_{0}, t\right)=f_{1} \int_{0}^{t} \mu_{x_{0}}(t-s) d s, \quad 0 \leq t \leq t_{1} .
$$

Choosing $L_{p}$ norm from 0 to $t_{1}$, we obtain

$$
\|u\|_{L_{p}\left(0, t_{1}\right)}=\left|f_{1}\right|\left\|\int_{0}^{t} \mu_{x_{0}}(t-s) d s\right\|_{L_{p}\left(0, t_{1}\right)} .
$$

Thus, we can see that

$$
\left|f_{1}\right| \leq C_{1}\|u\|_{L_{p}\left(0, t_{1}\right)},
$$

where $C_{1}>\left(\left\|\int_{0}^{t} \mu_{x_{0}}(t-s) d s\right\|_{L_{p}\left(0, t_{1}\right)}\right)^{-1}$ is a constant.

For $t_{1} \leq t \leq t_{2}$, we can see that

$$
\begin{aligned}
& u\left(x_{0}, t\right)=\int_{0}^{t} \mu_{x_{0}}(t-s) f(s) d s \\
& =f_{1} \int_{0}^{t_{1}} \mu_{x_{0}}(t-s) d s+f_{2} \int_{t_{1}}^{t} \mu_{x_{0}}(t-s) d s, \\
& u\left(x_{0}, t\right)-f_{1} \int_{0}^{t_{1}} \mu_{x_{0}}(t-s) d s=f_{2} \int_{t_{1}}^{t} \mu_{x_{0}}(t-s) d s .
\end{aligned}
$$

Then, we can obtain

$$
\begin{aligned}
\left|f_{2}\right| \cdot & \left\|\int_{t_{1}}^{t} \mu_{x_{0}}(t-s) d s\right\|_{L_{p}\left(t_{1}, t_{2}\right)} \\
= & \left\|u\left(x_{0}, t\right)-f_{1} \int_{0}^{t_{1}} \mu_{x_{0}}(t-s) d s\right\|_{L_{p}\left(t_{1}, t_{2}\right)} \\
\leq & \left\|u\left(x_{0}, t\right)\right\|_{L_{p}\left(t_{1}, t_{2}\right)} \\
& +\left|f_{1}\right|\left\|\int_{0}^{t_{1}} \mu_{x_{0}}(t-s) d s\right\|_{L_{p}\left(t_{1}, t_{2}\right)} \\
\leq & \left\|u\left(x_{0}, t\right)\right\|_{L_{p}\left(t_{1}, t_{2}\right)}+C_{2}\left\|u\left(x_{0}, t\right)\right\|_{L_{p}\left(0, t_{1}\right)} \\
\leq & C_{3}\left\|u\left(x_{0}, t\right)\right\|_{L_{p}\left(0, t_{2}\right)} .
\end{aligned}
$$

Then

$$
\left|f_{2}\right| \leq C\left\|u\left(x_{0}, t\right)\right\|_{L_{p}\left(0, t_{2}\right)} .
$$

Therefore, in the case of $t_{i}-t_{i-1} \geq c>0, i=1,2, \ldots, I$ and (20), we get

$$
\|f\|_{L_{p}\left(0, t_{2}\right)} \leq C\left\|u\left(x_{0}, t\right)\right\|_{L_{p}\left(0, t_{2}\right)} .
$$

Continuing this argument until $t_{i}=T$, we get the proof of this theorem.

\section{Numerical Scheme}

For problem (1), if $u\left(x_{0}, t\right)$ is given, we need to reconstruct function $f(t)$.

Consider an auxiliary problem:

$$
\begin{aligned}
u_{t} & =\Delta u+\phi(x) \quad(x, t) \in \Omega \times(0, T), \\
u(x, 0) & =0 \quad x \in \Omega, \\
u(x, t) & =0 \quad(x, t) \in \partial \Omega \times(0, T) .
\end{aligned}
$$

Actually, we can get the solution of problem (25) and suppose that $\tilde{u}$ is the solution. Then,

$$
\widetilde{u}(x, t)=\int_{0}^{t} \int_{\Omega} K(x, y, t-s) \phi(y) d y d s .
$$

Given data $u\left(x_{0}, t_{i}\right), i=0,1,2, \ldots, N$, where $0=t_{0}<$ $t_{1}<\cdots<t_{N}=T$, then, for every $t_{i}$, we have

$$
u\left(x_{0}, t_{i}\right)=\int_{0}^{t_{i}} \int_{\Omega} K\left(x_{0}, y, t_{i}-s\right) f(s) \phi(y) d y d s
$$

$$
i=0,1,2, \ldots, N \text {. }
$$
then

We suppose that $f(t)=f_{i}, t \in\left[t_{i-1}, t_{i}\right], i=1,2, \ldots, N$;

$$
\begin{aligned}
& u\left(x_{0}, t_{i}\right)=\int_{0}^{t_{i}} \int_{\Omega} K\left(x_{0}, y, t_{i}-s\right) f(s) \phi(y) d y d s \\
& =\sum_{j=1}^{i} f_{j} \int_{t_{j-1}}^{t_{j}} \int_{\Omega} K\left(x_{0}, y, t_{i}-s\right) \phi(y) d y d s \\
& =f_{1} \int_{0}^{t_{i}} \int_{\Omega} K\left(x_{0}, y, t_{i}-s\right) \phi(y) d y d s \\
& +\sum_{j=2}^{i}\left(f_{j}-f_{1}\right) \int_{t_{j-1}}^{t_{j}} \int_{\Omega} K\left(x_{0}, y, t_{i}-s\right) \phi(y) d y d s \\
& =f_{1} \tilde{u}\left(x_{0}, t_{i}\right)+\left(f_{2}-f_{1}\right) \\
& \cdot \int_{t_{1}}^{t_{i}} \int_{\Omega} K\left(x_{0}, y, t_{i}-s\right) \phi(y) d y d s \\
& +\sum_{j=3}^{i}\left(f_{j}-f_{1}-\left(f_{2}-f_{1}\right)\right) \\
& \cdot \int_{t_{j-1}}^{t_{j}} \int_{\Omega} K\left(x_{0}, y, t_{i}-s\right) \phi(y) d y d s=f_{1} \widetilde{u}\left(x_{0}, t_{i}\right) \\
& +\left(f_{2}-f_{1}\right) \\
& \cdot \int_{0}^{t_{i}-t_{1}} \int_{\Omega} K\left(x_{0}, y, t_{i}-t_{1}-s\right) \phi(y) d y d s \\
& +\sum_{j=3}^{i}\left(f_{j}-f_{2}\right) \int_{t_{j-1}}^{t_{j}} \int_{\Omega} K\left(x_{0}, y, t_{i}-s\right) \phi(y) d y d s \\
& =\cdots=f_{1} \tilde{u}\left(x_{0}, t_{i}\right) \\
& +\sum_{j=2}^{i}\left(f_{j}-f_{j-1}\right) \tilde{u}\left(x_{0}, t_{i}-t_{j-1}\right) \text {. }
\end{aligned}
$$




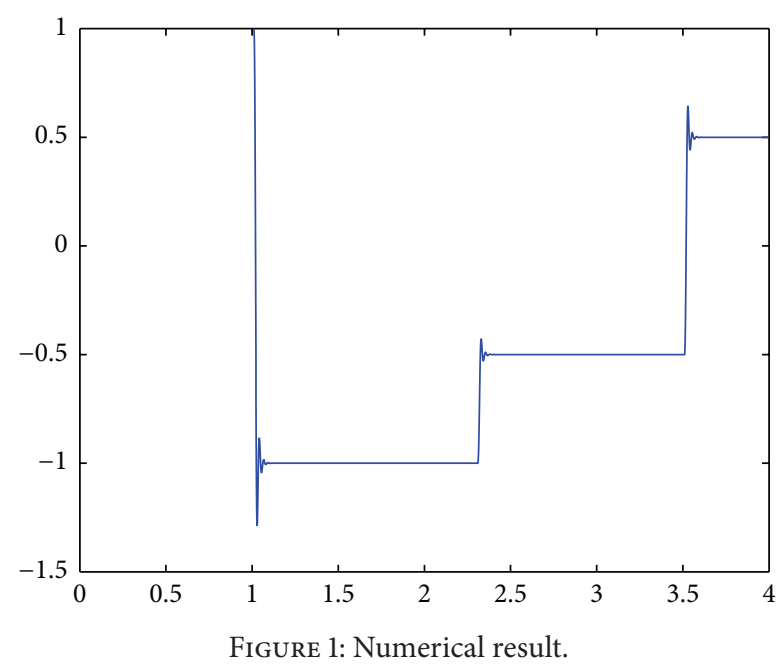

Thus, we can use the above formula to get the discrete data for function $f(t)$.

Example 6. We choose $f(t)$ as the following function:

$$
f(t)= \begin{cases}1, & t \in[0,1) \\ -1, & t \in[1,2.3) \\ -0.5, & t \in[2.3,3.5) \\ 0.5, & t \in[3.5,4] .\end{cases}
$$

Choose $\Omega=(0,2), x_{0}=1.5, \phi(x)=1, x \in(0,1)$, and $\phi(x) \equiv 0$ outside interval $(0,1)$. Given data $u\left(x_{0}, t_{i}\right), i=$ $1,2, \ldots, N$. Then, we can get the numerical approximation of $f(t)$ using the following formula:

$$
\begin{aligned}
& u\left(x_{0}, t_{i}\right)=f_{1} \tilde{u}\left(x_{0}, t_{i}\right) \\
& +\sum_{j=2}^{i}\left(f_{j}-f_{1}\right) \tilde{u}\left(x_{0}, t_{i}-t_{j-1}\right), \\
& \quad i=1,2, \ldots, N .
\end{aligned}
$$

And the numerical result is as shown in Figure 1.

Figure 1 is the numerical result and Figure 2 is the exact function.

Figure 3 shows the error between the numerical result and exact solution.

Example 7. Now, we will give a numerical result for the $2 \mathrm{D}$ case. Suppose the problem is also (1), and the domain $\Omega=$ $(-1,1) \times(-1,1)$, and

$$
\begin{aligned}
& \phi(x)= \begin{cases}1, & |x| \leq 0.5 \\
0, & x \in \Omega, \quad|x|>0.5\end{cases} \\
& f(t)= \begin{cases}1, & t \in[0,0.8) \\
0, & t \in[0.8,2.2) \\
-2, & t \in[2.2,3] .\end{cases}
\end{aligned}
$$
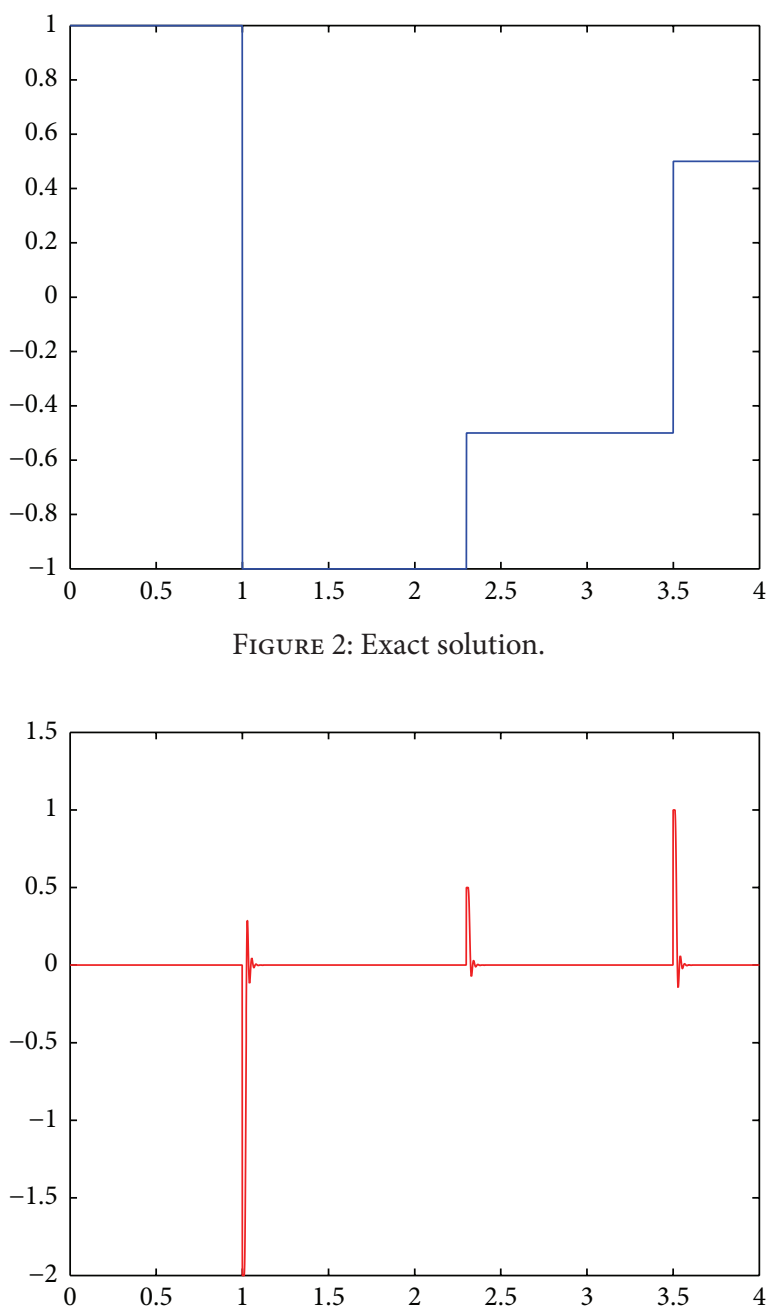

Figure 3: The error between numerical result and exact solution.

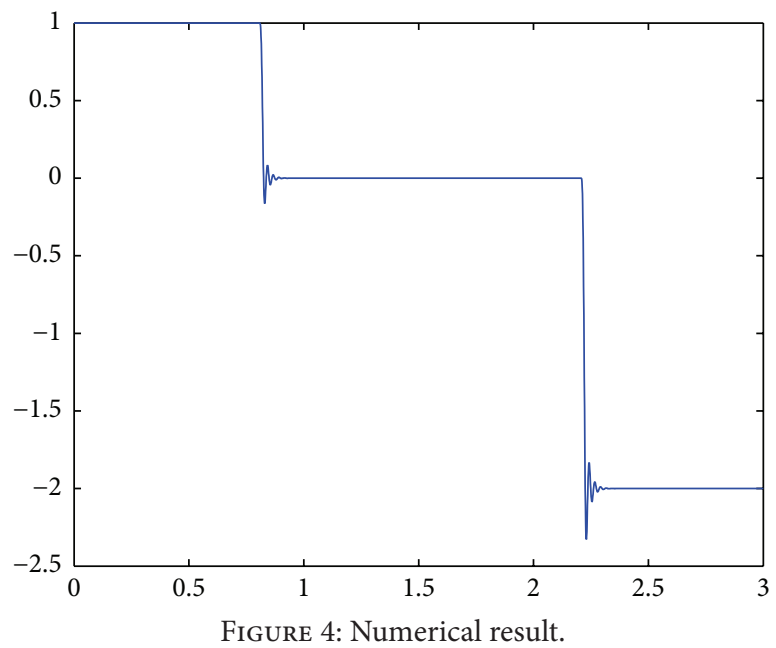

Let $x_{0}=(0.7,0.8)$ and $T=3$, and the numerical result is as shown in Figure 4.

Figure 4 is the numerical result and Figure 5 is the exact function. 

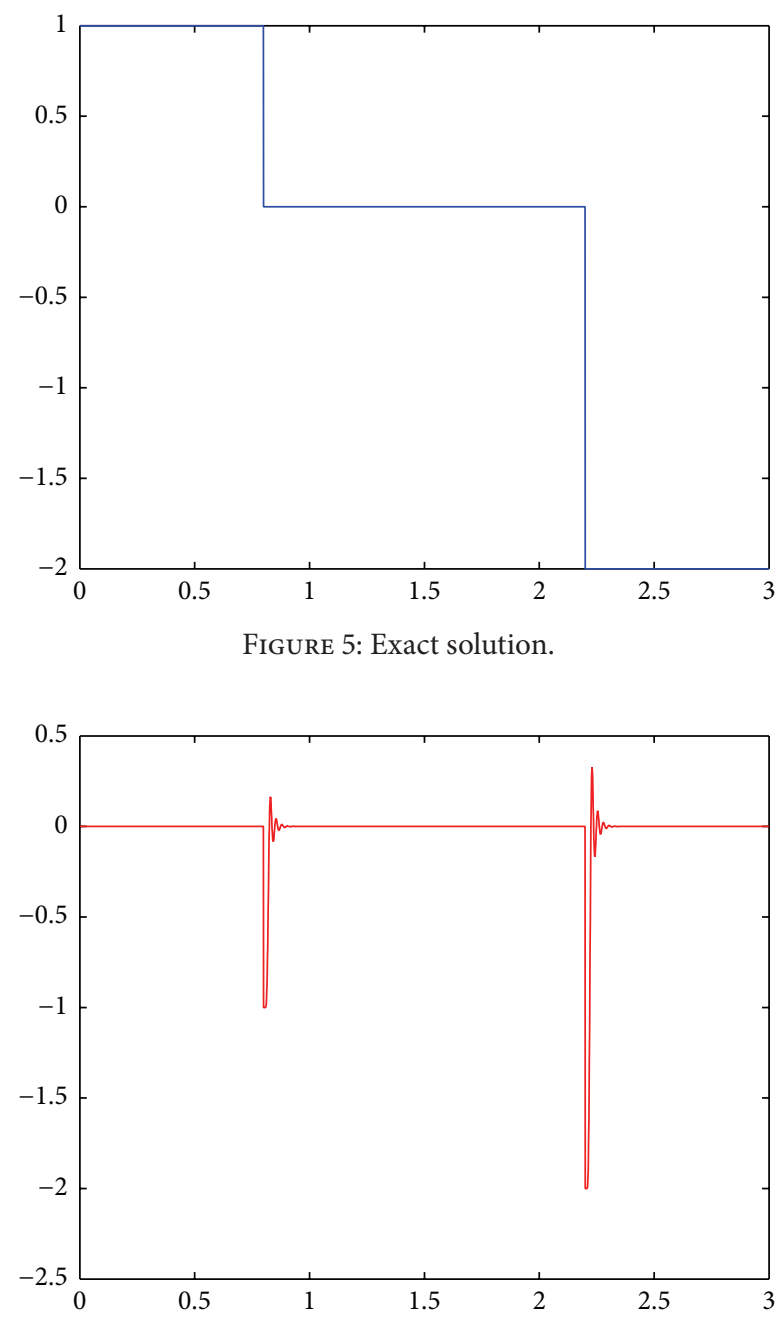

FIGURE 6: The error between numerical result and exact solution. result.

Figure 6 is the error between exact $f$ and the numerical

\section{Competing Interests}

The author declares that there are no competing interests regarding the publication of this paper.

\section{Acknowledgments}

This work was supported by the Natural Science Foundation of China Grant no. 11371231 and Grant no. 11071148 and the Natural Science Foundation of Shandong Province, China, Grant no. ZR2011AQ014.

\section{References}

[1] J. R. Cannon and P. DuChateau, "Structural identification of an unknown source term in a heat equation," Inverse Problems, vol. 14, no. 3, pp. 535-551, 1998.

[2] L. Yan, C.-L. Fu, and F.-L. Yang, "The method of fundamental solutions for the inverse heat source problem," Engineering
Analysis with Boundary Elements, vol. 32, no. 3, pp. 216-222, 2008.

[3] S. Chantasiriwan, "Methods of fundamental solutions for timedependent heat conduction problems," International Journal for Numerical Methods in Engineering, vol. 66, no. 1, pp. 147-165, 2006.

[4] L. Yan, F.-L. Yang, and C.-L. Fu, "A meshless method for solving an inverse spacewise-dependent heat source problem," Journal of Computational Physics, vol. 228, no. 1, pp. 123-136, 2009.

[5] M. N. Ahmadabadi, M. Arab, and F. M. M. Ghaini, "The method of fundamental solutions for the inverse space-dependent heat source problem," Engineering Analysis with Boundary Elements, vol. 33, no. 10, pp. 1231-1235, 2009.

[6] T. Johansson and D. Lesnic, "Determination of a spacewise dependent heat source," Journal of Computational and Applied Mathematics, vol. 209, no. 1, pp. 66-80, 2007.

[7] A. Farcas and D. Lesnic, "The boundary-element method for the determination of a heat source dependent on one variable," Journal of Engineering Mathematics, vol. 54, no. 4, pp. 375-388, 2006.

[8] B. T. Johansson and D. Lesnic, "A variational method for identifying a spacewise-dependent heat source," IMA Journal of Applied Mathematics, vol. 72, no. 6, pp. 748-760, 2007.

[9] B. T. Johansson and D. Lesnic, "A procedure for determining a spacewise dependent heat source and the initial temperature," Applicable Analysis, vol. 87, no. 3, pp. 265-276, 2008.

[10] B. Jin and L. Marin, "The method of fundamental solutions for inverse source problems associated with the steady-state heat conduction," International Journal for Numerical Methods in Engineering, vol. 69, no. 8, pp. 1570-1589, 2007.

[11] F. Wang, W. Chen, and L. Ling, "Combinations of the method of fundamental solutions for general inverse source identification problems," Applied Mathematics and Computation, vol. 219, no. 3, pp. 1173-1182, 2012.

[12] M. Mierzwiczak and J. A. Kolodziej, "Application of the method of fundamental solutions and radial basis functions for inverse transient heat source problem," Computer Physics Communications, vol. 181, no. 12, pp. 2035-2043, 2010.

[13] M. Mierzwiczak and J. A. Kołodziej, "Application of the method of fundamental solutions with the Laplace transformation for the inverse transient heat source problem," Journal of Theoretical and Applied Mechanics, vol. 50, no. 4, pp. 1011-1023, 2012.

[14] E. G. Savateev, "On problems of determining the source function in a parabolic equation," Journal of Inverse and Ill-Posed Problems, vol. 3, no. 1, pp. 83-102, 1995.

[15] D. D. Trong, N. T. Long, and P. N. Alain, "Nonhomogeneous heat equation: identification and regularization for the inhomogeneous term," Journal of Mathematical Analysis and Applications, vol. 312, no. 1, pp. 93-104, 2005.

[16] A. Shidfar, B. Jazbi, and M. Alinejadmofrad, "Inverse estimation of the pulse parameters of a time-varying laser pulse to obtain desired temperature at the material surface," Optics and Laser Technology, vol. 44, no. 6, pp. 1675-1680, 2012.

[17] S. Saitoh, V. K. Tuan, and M. Yamamoto, "Reverse convolution inequalities and applications to inverse heat source problems," Journal of Inequalities in Pure and Applied Mathematics, vol. 3, no. 5, article 80, 2002.

[18] S. Itô, Diffusion Equations, AMS, Providence, RI, USA, 1992. 


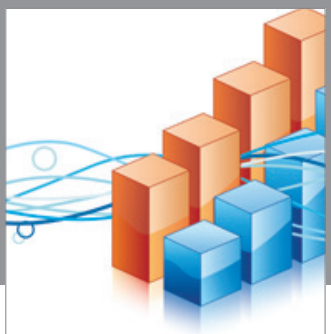

Advances in

Operations Research

vatem alat4

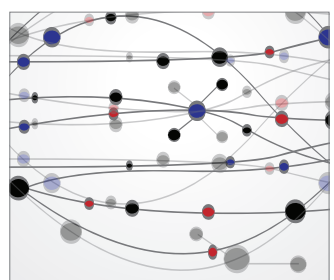

\section{The Scientific} World Journal
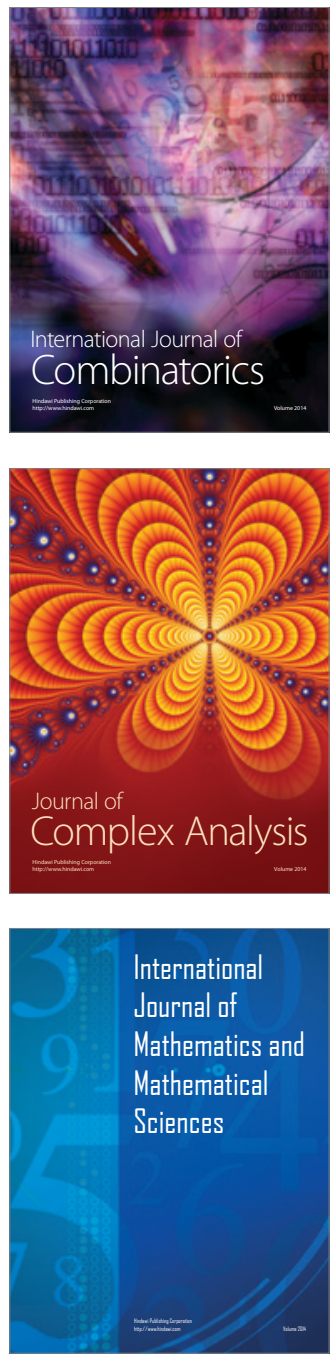
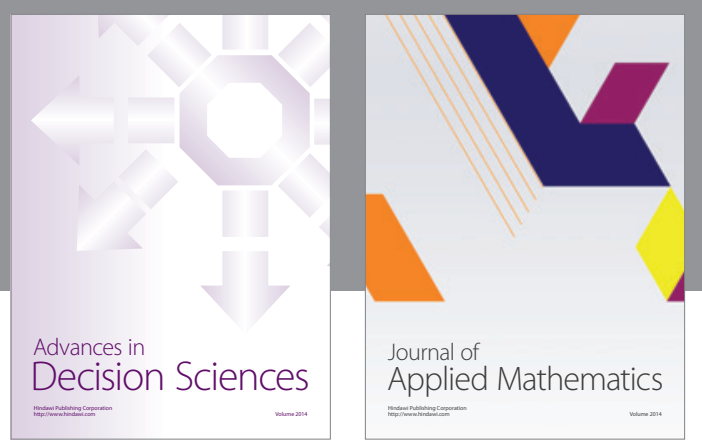

Algebra

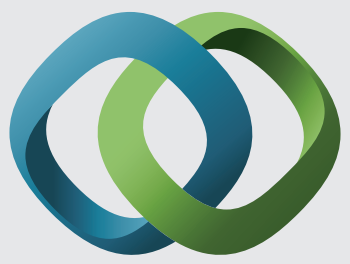

\section{Hindawi}

Submit your manuscripts at

http://www.hindawi.com
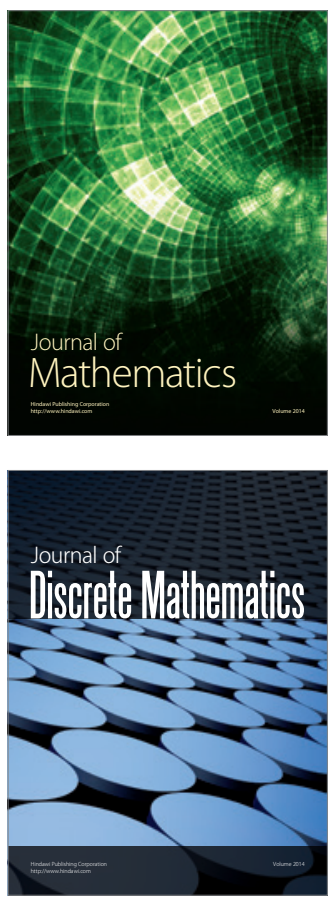

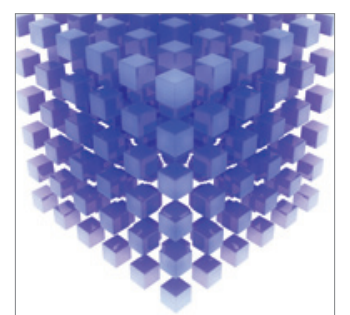

Mathematical Problems in Engineering
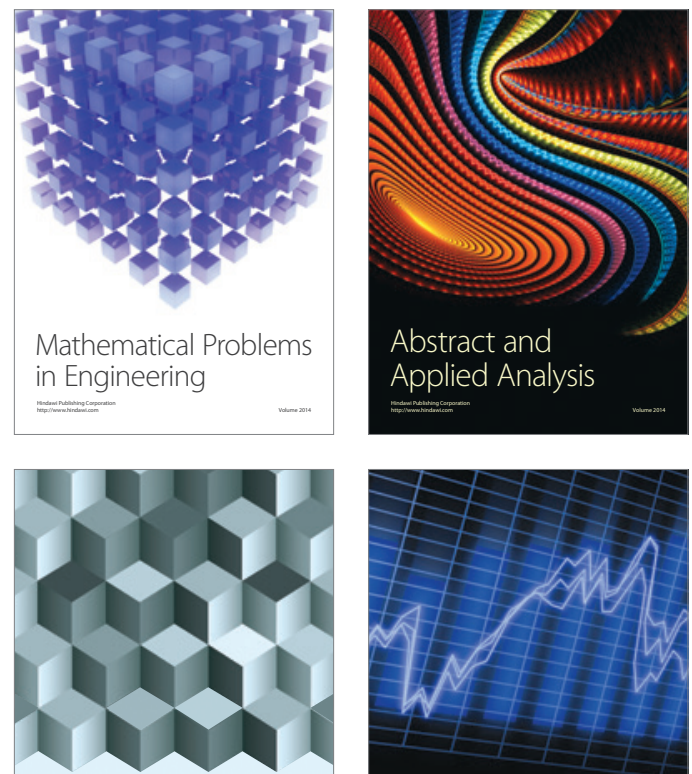

Journal of

Function Spaces

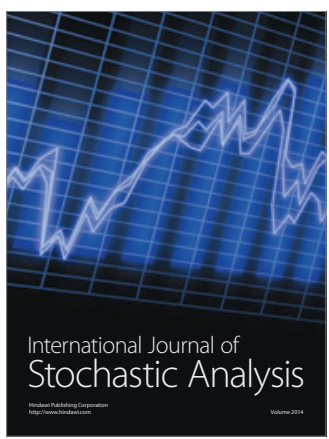

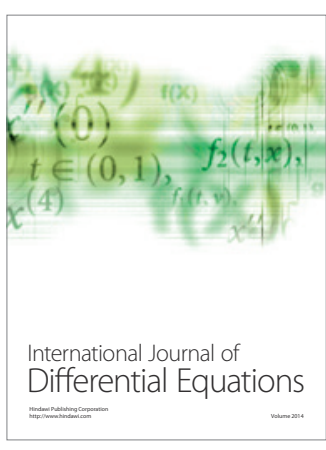
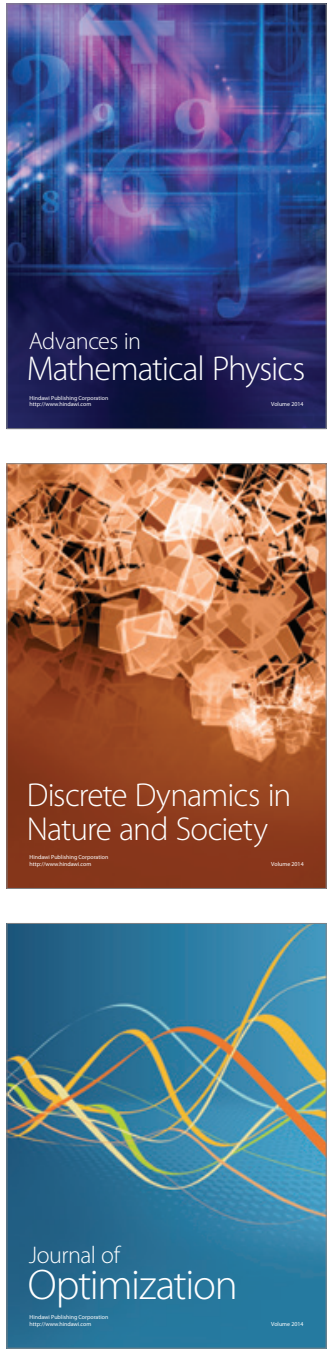